\title{
EDITORIAL
}

\section{Bronchoscopic lung volume reduction for emphysema: where next?}

\author{
Pallav L. Shah and Nicholas S. Hopkinson
}

E mphysema is an irreversible disease and although patients derive significant benefit from inhaled therapies [1] and pulmonary rehabilitation [2], many remain significantly disabled. The National Emphysema Treatment Trial of lung volume reduction clearly showed that in appropriately selected patients it is possible to improve lung function, exercise capacity, quality of life and survival $[3,4]$. A search for new approaches to produce lung volume reduction, either avoiding the morbidity and mortality potentially associated with surgery or aimed at patients who do not meet the criteria for lung volume reduction surgery (LVRS), has been underway. Over the last decade, since the first report of bronchoscopic lung volume reduction in patients with emphysema [5], there has been a significant expansion in research and investment into this area [6-13]. Approaches have included the placement of endobronchial valves and RePneu ${ }^{\mathrm{TM}}$ endobronchial coils (PneumRX, Mountain View, CA, USA) [11], as well as biological agents to induce scarring [9] and airway bypass approaches, either endobronchially [13] or transpleurally [12].

Endobronchial valves are intended to prevent air reaching the target area of the lung, leading to resorption of air and reduction of gas trapping. The valve mechanism also allows air to leave and prevents the acute local hyperinflation reported with simple airway-blocking devices [14]. A limitation to this approach is the presence of interlobar collateral ventilation. Where this occurs, occlusion of all segmental bronchi will not isolate a lobe, because air can still enter through pathological channels from adjacent damaged lung.

Following initial case series [5, 7, 15], data from the US arm of the first randomised controlled trial of endobronchial valves, the Endobronchial Valve for Emphysema Palliation Trial (VENT), was published in 2010 [10]. In that study, unilateral placement of Zephyr ${ }^{\mathrm{TM}}$ valves (Pulmonx, Neuchâtel, Switzerland) to achieve lobar occlusion produced small but statistically significant improvements in forced expiratory volume in $1 \mathrm{~s}$ (FEV1) (6.8\% difference) and 6-min walk distance (5.8\%). Although the whole-group response was disappointing (and insufficient for the US Food and Drug Administration to approve the device), a "responder" phenotype could be identified: patients with intact interlobar fissures on computed

NIHR Respiratory Biomedical Research Unit at Royal Brompton and Harefield NHS Foundation Trust and Imperial College, London, and Chelsea and Westminster Hospital, London, UK.

CORRESPONDENCE: P.L. Shah, Royal Brompton Hospital, Sydney Street, London SW3 6NP, UK. E-mail: pallav.shah@imperial.ac.uk tomography (CT) had a $17.9 \%$ improvement in FEV1 at 12 months compared with a $2.8 \%$ increase where fissures were incomplete. Greater heterogeneity, defined as the difference in CT emphysema score between the target lobe and the adjacent lobe, was associated with greater improvements both in FEV1 and exercise capacity. Both these parameters are likely to represent the absence of collateral ventilation.

In this issue of the European Respiratory Journal (ERJ), HERTH et al. [16] present the results of the European VENT study. These were broadly similar to those from the USA. There were again modest improvements in FEV1, exercise capacity and quality of life in the treatment group as a whole compared with controls. However, in patients with intact interlobar fissures (roughly one-third of the total) and satisfactory valve placement, the target lobe volume decreased by a mean \pm SD of $80 \pm 30 \%$ and FEV1 increased by $26 \%$. Two-thirds of patients exceeded the minimum clinically important difference (MCID) for the latter measurement at 12 months (i.e. $>15 \%$ improvement in FEV1).

In contrast, also in the current issue, a European study with the Intrabronchial Valve ${ }^{\mathrm{TM}}$ (IBV; Olympus, Hamburg, Germany) showed no clinical benefit [17]. NINANE et al. [17] performed a randomised, single-blind study with the IBV using a strategy of bilateral treatment but with incomplete occlusion of the target lobes. 37 patients received valves and 36 had a sham bronchoscopy. After 3 months, there was no significant difference in lung function, breathlessness or health-related quality of life assessed using the St George's respiratory questionnaire (SGRQ) and Short Form-36. A composite primary end-point that combined SGRQ and CT-determined lobar volume changes was met in eight patients in the treatment arm and none in the control arm, but in the absence of improvements in lung function parameters, these lobar volume changes must be of limited significance. The important finding of the study, therefore, is that a nonlobar occlusion approach is ineffective when endobronchial valves are used for the treatment of heterogeneous emphysema. This is perhaps unsurprising given that collateral ventilation appears to be such an important limiting factor. Leaving a segmental airway open is likely to have the same effect where intralobar (i.e. between segments) rather than interlobar collateral ventilation exists. NINANE et al. [17] are to be commended for using a sham bronchoscopy. This highlighted a significant placebo effect, with both arms having an $\sim 4$-point improvement in SGRQ, the level considered to be the MCID for the test [18]. Although CT lung volume changes are informative, it is surprising that NINANE et al. [17] did not opt for a lung function or exercise 
capacity parameter with a defined level of clinical significance. In addition, the use of a co-primary end-point that cannot be blinded ( $\mathrm{CT}$ volume reduction) requires caution as the automated software for measuring lobar volumes requires a radiologist to check that the correct areas have been selected and the presence of valves on the CT scan would be obvious at this stage. Taken together, these studies $[16,17]$ provide further validation to the concept that "lobar exclusion", defined as appropriately placed valves in a lobe with intact interlobar fissures, is necessary for endobronchial valve therapy to be effective.

Also in this issue of the ERJ are combined data from two studies using unilateral bronchoscopic thermal vapour ablation (BTVA) to achieve volume reduction [19]. SNELL et al. [19] report outcomes for 44 patients with heterogeneous emphysema treated in two open-label studies (combined data set) with endobronchial application of steam. The application of thermal energy leads to focal inflammation that progresses to fibrosis and contraction of lung tissue. A potential advantage of BTVA is that success is independent of collateral ventilation, but the technique has the disadvantage of being irreversible. These early results are encouraging, with a satisfactory safety profile and significant improvements in lung function, exercise capacity and SGRQ at 6 months, although these must be interpreted with caution in the absence of a control group. A challenge for the design of future controlled studies will be that the procedure generates a reaction similar to an acute exacerbation of chronic obstructive pulmonary disease (COPD), which will make blinding participants to treatment allocation difficult.

There is increasing evidence that COPD needs to be considered in terms of specific disease phenotypes [20]. The data on survival benefit with LVRS in selected patients now mandates systematic assessment of patients to identify those with the appropriate characteristics: heterogeneous emphysema with poor exercise capacity [3]. In practice, this approach should include routine assessment of the pattern of emphysema by CT as well as gas transfer measurement in all patients with Medical Research Council dyspnoea scores of 4 or 5 and Global Initiative for Chronic Obstructive Lung Disease stage III or IV disease unless there are obvious comorbidities precluding surgery, with review by a multidisciplinary team including chest physicians, surgeons and radiologists, as is already the case for the management of lung cancer. The role of bronchoscopic techniques in different emphysema phenotypes will continue to evolve and is likely to remain complex as new technologies are developed, new data emerge from clinical trials and patient characteristics that determine the response to a particular intervention become more clearly defined. There is, however, increasing evidence that endobronchial valves can be effective where lobar exclusion is achieved and also encouraging data point to a survival advantage where lobar atelectasis occurs [6]. For commissioners of healthcare to accept these techniques, a consistent response will be necessary to allow for a realistic assessment of the cost/benefit ratio. The development of the Chartis ${ }^{\mathrm{TM}}$ system (Pulmonx), which uses a flow sensor incorporated into a balloon catheter to gauge the presence or absence of collateral ventilation [8], may allow further refinement in the selection of patients for treatment and prospective controlled studies are needed to confirm that a high response-rate group can be identified a priori.

For patients where endobronchial valves are not indicated because of incomplete fissures, another mechanical option, the endobronchial coil, which acts by tensioning the lung to prevent airway collapse, may become an appropriate option [11]. The failure of the Exhale Airway Stents for Emphysema study of airway bypasses to show sustained benefit is a setback for patients with homogenous emphysema, though the shortterm reduction in gas-trapping achieved suggests that it could be a worthwhile approach if it were possible to develop stents that did not become occluded [13]. Further work is needed to clarify the effectiveness of tissue destructive techniques, such as BTVA and the use of biological agents [9], but these too show promise. A key issue for future work in this area will be to move towards a consensus about end-points to ensure that these are physiologically appropriate and patient-relevant, in order to aid comparison of treatments and commissioning decisions.

\section{STATEMENT OF INTEREST}

Statement of interest for P.L. Shah and N.S. Hopkinson can be found at www.erj.ersjournals.com/site/misc/statements.xhtml

\section{REFERENCES}

1 National Institute for Clinical Excellence. Management of chronic obstructive pulmonary disease in adults in primary and secondary care (partial update). This guideline partially updates and replaces NICE clinical guideline 12. Clinical guidelines CG101. http:// guidance.nice.org.uk/CG101 Date last accessed: March 22, 2012. Date last updated: January 18, 2012.

2 Dodd JW, Hogg L, Nolan J, et al. The COPD assessment test (CAT): response to pulmonary rehabilitation. A multicentre, prospective study. Thorax 2011; 66: 425-429.

3 Criner GJ, Cordova F, Sternberg AL, et al. The National Emphysema Treatment Trial (NETT): part II: lessons learned about lung volume reduction surgery. Am J Respir Crit Care Med 2011; 184: 881-893.

4 Fishman A, Martinez F, Naunheim K, et al. A randomized trial comparing lung-volume-reduction surgery with medical therapy for severe emphysema. N Engl J Med 2003; 348: 2059-2073.

5 Toma TP, Hopkinson NS, Hillier J, et al. Bronchoscopic volume reduction with valve implants in patients with severe emphysema. Lancet 2003; 361: 931-933.

6 Hopkinson NS, Kemp SV, Toma TP, et al. Atelectasis and survival after bronchoscopic lung volume reduction for COPD. Eur Respir J 2011; 37: 1346-1351.

7 Hopkinson NS, Toma TP, Hansell DM, et al. Effect of bronchoscopic lung volume reduction on dynamic hyperinflation and exercise in emphysema. Am J Respir Crit Care Med 2005; 171: 453-460.

8 Gompelmann D, Eberhardt R, Michaud G, et al. Predicting atelectasis by assessment of collateral ventilation prior to endobronchial lung volume reduction: a feasibility study. Respiration 2010; 80: 419-425.

9 Herth FJ, Gompelmann D, Stanzel F, et al. Treatment of advanced emphysema with emphysematous lung sealant (AeriSeal ${ }_{\mathbb{}}$ ). Respiration 2011; 82: 36-45.

10 Sciurba FC, Ernst A, Herth FJF, et al. A randomized study of endobronchial valves for advanced emphysema. $N$ Engl J Med 2010; 363: 1233-1244.

11 Slebos DJ, Klooster K, Ernst A, et al. Bronchoscopic lung volume reduction coil treatment of patients with severe heterogeneous 
emphysema. Chest 2011; [Epub ahead of print DOI: 10.1378/ chest.11-0730].

12 Moore AJ, Cetti E, Haj-Yahia S, et al. Unilateral extrapulmonary airway bypass in advanced emphysema. Ann Thorac Surg 2010; 89: 899-906.

13 Shah PL, Slebos DJ, Cardoso PFG, et al. Bronchoscopic lung-volume reduction with Exhale airway stents for emphysema (EASE trial): randomised, sham-controlled, multicentre trial. Lancet 2011; 378: 997-1005.

14 Sabanathan S, Richardson J, Pieri-Davies S. Bronchoscopic lung volume reduction. J Cardiovasc Surg (Torino) 2003; 44: 101-108.

15 Wan IY, Toma TP, Geddes DM, et al. Bronchoscopic lung volume reduction for end-stage emphysema: report on the first 98 patients. Chest 2006; 129: 518-526.
16 Herth FJF, Noppen M, Valipour A, et al. Efficacy predictors of lung volume reduction with Zephyr valves in a European cohort. Eur Respir J 2012; 39: 1334-1342.

17 Ninane V, Geltner C, Bezzi M, et al. Multicentre European study for the treatment of advacned emphysema with bronchial valves. Eur Respir J 2012; 39: 1319-1325.

18 Jones PW. Health status measurement in chronic obstructive pulmonary disease. Thorax 2001; 56: 880-887.

19 Snell G, Herth FJF, Hopkins P, et al. Bronchoscopic thermal vapour ablation therapy in the management of heterogeneous emphysema. Eur Respir J 2012; 39: 1326-1333.

20 Agusti A, Calverley P, Celli B, et al. Characterisation of COPD heterogeneity in the ECLIPSE cohort. Respir Res 2010; 11: 122. 